\title{
RECURRENT LARYNGEAL NERVE PALSY AFTER CERVICAL SPINE SURGERY CAN BE PREVENTED
}

\author{
(1) Hüseyin DOǦU1', (1) Halil CAN² \\ ${ }^{1}$ Atlas University, Department of Neurosurgery, Istanbul, Turkey \\ ${ }^{2}$ Biruni University, Department of Neurosurgery, Istanbul, Turkey
}

Objective: To prevent postoperative laryngeal nerve palsy in patients undergoing anterior cervical spine surgery (ACSS).

Materials and Methods: A retrospective study was conducted with data from 643 patients who underwent ACSS between 2006 and 2019. Of these patients, 125 who underwent only ACSS served as group I; the other 518, who underwent retractor loosening and displacement along with ACSS, served as group II. The two groups were compared, and paralyzed patients were compared with the general community. In the 518 patients in group II, the Cloward retractor was loosened every 15 minutes, which resulted in a slight change in its position. This protocol was unlike the standard procedure. The retractor was left loose during copy scopy.

Results: The majority (58\%) of the patients were female, and average age was 47 years (range: 24 to 75 years). The mean duration of surgery was 152 minutes in group I and 162 minutes in group II. Transient laryngeal nerve palsy developed postoperatively in three patients (2.4\%) of group I but no patients in group II. No significant difference was observed in terms of surgical level, duration of surgery, age, gender, or comorbid conditions.

Conclusion: Retractor loosening and displacement for every 15 minutes during ACSS helps prevent postoperative recurrent laryngeal nerve palsy. Keywords: Recurrent laryngeal nerve palsy, cervical spine, spine surgery

\section{INTRODUCTION}

Anterior cervical spine surgery (ACSS) is the intervention most commonly preferred for the management of cervical spine disease; the surgical techniques are well documented. R. A. Robinson and G. W. Smith, Albert Dereymaeker and Joseph C. Mulier, and Ralph B. Cloward in 1950s were the first to describe the surgical techniques for ACSS; since then, the techniques have undergone several modifications and become widely used. ACSS enables efficient management of spinal disorders such as cervical stenosis, cervical myelopathy, and cervical radiculopathy. It provides easy access to the vertebrae and produces highly satisfactory surgical outcomes in the majority of $\operatorname{cases}^{(1)}$, and it remains the "gold standard" for the management of various spinal disorders. Anterior cervical discectomy and fusion, in particular, has high success rates and low complication rates.

Despite the advantages of ACSS, postoperative complications occur. The most common complication after ACSS is recurrent laryngeal nerve palsy (RLNP), followed by vocal fold paralysis or vocal cord paralysis ${ }^{(2)}$. RLNP results from ischemia caused by surgical pressure, neuropraxia caused by overstretching, and edema that results from perioperative trauma ${ }^{(3)}$. We evaluated the efficacy of retractor loosening and displacement during surgery in preventing postoperative RLNP.

\section{MATERIALS AND METHODS}

Data from 643 patients who underwent ACSS between 2006 and 2019 were studied retrospectively. Of these patients, 125, who served as group I, underwent standard ACSS; the other 518, who served as group II, underwent ACSS and retractor loosening and displacement every 15 minutes; that is, the retractor was repositioned slightly during ACSS. A member of the surgery team was assigned to remind the retractor to be repositioned. He or she used the stopwatch while making the timing. This procedure was repeated every 15 minutes, and the retractor was left loose during scopy shots. Finally, low cutoff pressure was not applied in both group I and group II. All operations were performed at the same hospital and by the same surgeon. The surgical outcomes measured were operation time, postoperative RLNP development, and characteristic of patients with RLNP in groups I and II. 


\section{RESULTS}

Of the total of 643 patients, 367 (57\%) were female and 276 (43\%) were male (Table 1). The average age of the patients with injury was 47.2 years, and mean duration of surgery in those patients was 148.79 minutes (Table 1 ). Of the 125 patients in group I, 76 (60.8\%) were female and 49 (39.2\%) were male. Of the 518 patients in group II, 291 (56.2\%) were female and 227 (43.8\%) were male (Table 1). There was no difference between these groups in average age (47 years), but the mean duration of surgery was significantly longer for group II (152.24 \pm 83.01 minutes), $\mathrm{p}=0.023$ (Table 2).

Of all 643 patients, three (2.4\%) patients had RLNP; all three were from group I (Table 3). The patients with RLNP were given nonsteriodal anti-inflammatory drugs and low dose corticosteroid as medical treatment. They were trained to prevent aspiration. All of the patients with RLNP recovered within 2 months without the need for a second surgery.

There were no significant differences in mean age and mean duration of surgery between group I and the total patient population (Table 4). The majority of patients [416 (64.7\%)] underwent one-level surgery; of these patients, 72 (57.6\%) were in group I and 344 (66.4\%) were in group II. (Table 5). The observed chi-square value was 4.477 and $p=0.107$, which indicated no association between the number of surgical levels and patient groups.

Groups were evaluated statistically in terms of gender (Table 1). The observed chi-square value was 0.878 and $p=0.349$, which indicated no association between gender and groups.

\section{DISCUSSION}

Cervical spinal disorders not only affect patients' health but also increase the economic burden on the individuals, families, and most of society. Most cervical diseases can be managed non-surgically, but early surgical intervention is recommended in cases of neurological impairment to improve its functional outcome $e^{(4,5)}$. Although the anterior approach of cervical surgery is most preferred by surgeons because of its high rates of clinical success, RLNP continues to be the major postoperative complication of ACSS that results from surgical pressure ${ }^{(3)}$.

Table 1. Associations between gender and groups.

\begin{tabular}{llll} 
& & Group & \\
\hline Gender & Group I $(\mathbf{n}=\mathbf{1 2 5})$ & Group II $(\mathbf{n}=\mathbf{5 1 8})$ & Total \\
\hline Male & $49(39.2 \%)$ & $227(43.8 \%)$ & $276(42.9 \%)$ \\
\hline Female & $76(60.8 \%)$ & $291(56.2 \%)$ & $367(57.1 \%)$ \\
\hline Total & $125(100.0 \%)$ & $518(100.0 \%)$ & $643(100.0 \%)$ \\
\hline
\end{tabular}

n: Number

Chi-square value: $0.878,0.349>p>0.05$

Table 2. Difference in mean age and duration of surgery between groups I and II

\begin{tabular}{llcll}
\hline \multirow{2}{*}{ Measure } & Group I $(\mathrm{n}=125)$ & & \\
\cline { 2 - 5 } & & Mean \pm standard deviation & & $\mathrm{p}$ \\
\hline Age (years) & $47.02 \pm 10.11$ & $47.30 \pm 10.66$ & -0.258 & 0.797 \\
\hline $\begin{array}{l}\text { Duration of surgery } \\
\text { (minutes) }\end{array}$ & $134.50 \pm 52.54$ & $152.24 \pm 83.01$ & -2.281 & $0.023^{*}$ \\
\hline${ }^{*} p<0.05$ & & & & \\
\hline
\end{tabular}

Table 3. Percentage of patients with paralysis in groups I and II

\begin{tabular}{llll}
\hline Measure & Total & Group I & Group II \\
\hline Cases & 643 & 125 & 518 \\
\hline Paralysis & 3 & $3(2.4 \%)$ & 0 \\
\hline
\end{tabular}

Table 4. Comparison of mean age and duration of surgery between patients with paralysis and total patient population

\begin{tabular}{llll}
\hline \multirow{2}{*}{ Measure } & \multicolumn{3}{c}{ Mean \pm standard deviation } \\
\cline { 2 - 4 } & Patients with paralysis $(n=3)$ & Total patient population $(n=643)$ & 0.138 \\
\hline Age (in years) & $56.33 \pm 18.58$ & $47.24 \pm 10.55$ & 0.099 \\
\hline Duration of surgery (minutes) & $74.00 \pm 15.10$ & $148.79 \pm 78.31$ & $p$ \\
\hline
\end{tabular}


Table 5. Number of surgical levels by group

\begin{tabular}{llll}
\hline \multirow{2}{*}{ Number of levels } & \multicolumn{2}{c}{ Group } & Total \\
\cline { 2 - 4 } & Group I & Group II & $416(64.7 \%)$ \\
\hline $\mathbf{1}$ & $72(57.6 \%)$ & $344(66.4 \%)$ & $173(26.9 \%)$ \\
\hline $\mathbf{2}$ & $43(34.4 \%)$ & $130(25.1 \%)$ & $54(8.4 \%)$ \\
\hline $\mathbf{3}$ & $10(8.0 \%)$ & $44(8.5 \%)$ & $643(100.0 \%)$ \\
\hline Total & $125(100.0 \%)$ & $518(100.0 \%)$ & \\
\hline
\end{tabular}

In a retrospective review, Kriskovich et al.(2) tested "whether controlling for endotracheal tube/laryngeal wall interactions by cervical retraction system [would] reduce symptomatic or asymptomatic RLNP or permanent paralysis." They found that during surgery, the retractor "moved the larynx against the shaft of the endotracheal tube," thereby compressing the "vulnerable intralaryngeal segment of the laryngeal nerve." They therefore recommended continuous monitoring of endotracheal tube cuff pressure and intermittent or temporary release after retractor placement to allow repositioning of the endotracheal tube within the larynx and to prevent further injury to the laryngeal nerve. Similar findings were reported by Cheung and $\operatorname{Luk}^{(3)}$ and Matgé( ${ }^{(6)}$, who recommended the use and temporary release of a sharp-toothed retractor to prevent anteromedial and anterolateral displacement, which could otherwise compress and damage the trachea and carotid artery, respectively. In our study, similar to both studies, our finding is that intermittent retractor relaxation decreases RLNP. Unlike other studies, we standardized retractor relaxation within a certain time interval and did not release cuff.

The literature indicates that the left-sided approach, followed by low endotracheal cuff pressure and intermittent release of retractors tension during ACSS when not required, helps reduce the incidence of postoperative RLNP. However, there are sparse data on how often retractor tension can be reduced and on whether repositioning of retractors intraoperatively can help reduce RLNP. Hence, this retrospective, observational study of 643 patients who underwent ACSS was proposed, in which the majority of patients underwent retractor loosening and displacement during ACSS. The surgical outcome measured was based on success rate, duration of operation procedure, postoperative RLNP occurrence, and characteristic of patients with RLNP.

Single- or multiple-level discectomy is performed in $\operatorname{ACSS}^{(7)}$, and as the number of levels increases, radiographic non-fusion increases, as do complication rates ${ }^{(8-10)}$. However, in this study, no statistical association was observed between multiple-level surgery and the occurrence of RLNP; all three patients with postoperative RLNP underwent only one-level surgery.

The ratio of male and female patients with RLNP in this study was 2:1; their mean age was 56.33 years. The mean duration of surgery in all three cases did not exceed the general average; these patients had not undergone neck surgery previously, and none had preoperative hoarseness. There were no etiological factors or diseases that could cause RLNP in patients. However, the surgical approach was right-sided at C5-6 and C6-7, which is a major risk factor for postoperative RLNP(11,12). There is a claim that the left-sided intervention is safer. In our series, leftsided surgery was performed in five cases. However, since the number is low, it is not included in the series.

\section{Study Limitations}

This study has all the limitations of a retrospective study, and so a multicenter, matched case-control prospective study with a larger cohort is necessary to validate the findings. Another limitation of our study is that the pressure applied to the retractor and endotracheal cuff pressure are not measured. It must be determined how much retractor and cuff pressure causes RLNP formation. In patients who develop RLNP, the etiological factors that may cause such as the anatomical structure of the neck should be investigated.

\section{CONCLUSION}

Laryngeal nerve palsy is one of the most common postoperative surgical complications of ACSS. It must be addressed immediately to prevent permanent vocal cord paralysis and associated lifelong disability. This study showed that loosening and displacement of the Cloward retractor every 15 minutes during ACSS helped prevent RLNP and improved the clinical success rate. Hence, a large multicenter, matched case-control study to confirm these findings is warranted.

\section{Ethics}

Ethics Committee Approval: Retrospective study. Informed Consent: Retrospective study.

\section{Authorship Contributions}

Surgical and Medical Practices: H.D., Concept: H.D., Design: H.D., Data Collection or Processing: H.D., H.C., Analysis or Interpretation: H.D., H.C., Literature Search: H.D., H.C., Writing: H.D.

Conflict of Interest: No conflict of interest was declared by the authors.

Financial Disclosure: The author declared that this study received no financial support. 


\section{REFERENCES}

1. Tasiou A, Giannis T, Brotis AG, Siasios I, Georgiadis I, Gatos H et al. Anterior cervical spine surgery-associated complications in a retrospective case-control study. J Spine Surg. 2017;3:444-59.

2. Kriskovich MD, Apfelbaum RI, Haller JR. Vocal fold paralysis after anterior cervical spine surgery: incidence, mechanism, and prevention of injury. Laryngoscope. 2000;110:1467-73.

3. Cheung JPY, Luk KD-K. Complications of Anterior and Posterior Cervical Spine Surgery. Asian Spine J. 2016;10:385-400.

4. Garg R, Rath GP, Bithal PK, Prabhakar H, Marda MK. Effects of retractor application on cuff pressure and vocal cord function in patients undergoing anterior cervical discectomy and fusion. Indian J Anaesth. 2010;54:292-5.

5. O'Dowd JK. Basic principles of management for cervical spine trauma. Eur Spine J. 2010;19(Suppl 1):18-22.

6. Matgé G. Approaches to Cervical Spine. In: Sindou M. (eds) Practical Handbook of Neurosurgery. Springer, Vienna 2009; pp:1141-1160.
7. Mayfield Clinic \& Spine Institute. Anterior cervical discectomy and Fusion [online] 2013. http://www.mayfieldclinic.com/PE-ACDF.htm\#. Vc8aTvlcjVJ. (Accessed February 10 2020).

8. Kreitz TM, Hollern DA, Padegimas EM, Schroeder GD, Kepler CK, Vaccaro AR et al. Clinical Outcomes After Four-Level Anterior Cervical Discectomy and Fusion. Global Spine J. 2018;8:776-83.

9. Morgan JP, Asfora WT. Extreme multi-level anterior cervical discectomy and fusion. S D Med. 2013;66:273-7.

10. Bum-Suk Yu, Farid Yudoyono, Seong Yi, Yoon Ha, Keung Nyun Kim, Do Heum Yoon et al. Analysis of Surgical Results According to the Number of Fused Levels in Anterior Cervical Discectomy and Fusion: A retrospective study. The Nerve. 2017;3:32-37.

11. Gokaslan ZL, Bydon M, De la Garza-Ramos R, Smith ZA, Hsu WK, Qureshi SA et al. Recurrent Laryngeal Nerve Palsy After Cervical Spine Surgery: A Multicenter AOSpine Clinical Research Network Study. Global Spine J. 2017;7(1 Suppl):53S-57S.

12. Nikandish R, Zareizadeh A, Motazedian S, Zeraatian S, Zakeri H, Ghaffarpasand F. Bilateral Vocal Cord Paralysis after Anterior Cervical Discectomy Following Cervical Spine Injury: A Case Report. Bull Emerg Trauma. 2013;1:43-5. 